\title{
ON A BOUNDEDNESS CONDITION FOR OPERATORS WITH A SINGLETON SPECTRUM
}

\author{
J. P. WILLIAMS
}

\begin{abstract}
For a bounded invertible linear operator $A$ let $\mathscr{B}_{A}$ consist of those operators $X$ for which $\sup \left\{\left\|A^{n} X A^{-n}\right\|: n>0\right\}>\infty$. It is shown that $B_{A}$ contains the ideal of compact operators if and only if $A$ is similar to a scalar multiple of a unitary operator. Also, if $A$ is invertible and either has a one-point spectrum or is positive definite then $\mathscr{B}_{A} \cap \mathscr{B}_{A^{-1}}$ is the commutant of $A$.
\end{abstract}

In [2] Deddens shows that if $A=\int \lambda d E_{\lambda}$ is a positive invertible operator on a separable Hilbert space $\mathcal{H}$ then the nest algebra associated with the nest $\{E[0, \lambda]: \lambda \geqslant 0\}$ coincides with the set $\mathscr{B}_{A}$ of operators $X$ for which $\sup \left\{\left\|A^{n} X A^{-n}\right\|: n \geqslant 0\right\}<\infty$. Conversely every nest algebra is a $\mathscr{B}_{A}$ for some $A$. His results suggest that the boundedness condition defining $\mathscr{B}_{A}$ is of interest for any invertible $A$.

The present paper has three goals, namely to decide when $\mathscr{B}_{A}$ contains the compact operators, to simplify the discussion of the case in which $\mathscr{G}_{A}=\{A\}^{\prime}$ with $\mathcal{H}$ finite dimensional, and to give a partial resolution of the same problem in the general case: $\mathscr{B}_{A} \cap \mathscr{B}_{A^{-1}}=\{A\}^{\prime}$ if the spectrum of $A$ is a singleton.

It is a pleasure to acknowledge an indirect conversation with A. L. Shields which put me onto a theorem of Cartwright (see [1, 10.2.1]) which was a major ingredient in the original proof of Lemma 2. The simple argument given below was inspired by a comment of J. A. Deddens about a detail of that proof. I am grateful to him for several conversations about the results of this note.

We begin by resolving a question raised in [2]. (The referee mentions that the same result has been obtained by J. Stampfli and also by D. Herrero by different methods.)

THEOREM 1. $\mathscr{B}_{A}$ contains all the compact operators for some operator $A$ if and only if $A$ is similar to a scalar multiple of a unitary operator.

Proof. Let $\alpha_{n}(X)=A^{n} X A^{-n}$ for $n \geqslant 0$. If $\sup \left\{\left\|\alpha_{n}(K)\right\|: n \geqslant 0\right\}<\infty$ for each compact operator $K$ then the linear transformations $\alpha_{n}$ are uniformly bounded on the Banach space of compact operators by the Banach-Steinhaus theorem and $\left\|\alpha_{n}\right\| \leqslant M$. But then if $f \otimes g$ denotes the rank one operator that takes $h \in \mathcal{H}$ to $(h, g) f$ we have $\left\|A^{n} f\right\|\left\|A^{*-n} g\right\|=\left\|A^{n} f \otimes A^{*-n} g\right\|=$ $\left\|\alpha_{n}(f \otimes g)\right\| \leqslant M$ for all unit vectors $f$ and $g$. This gives $\left\|A^{n}\right\|\left\|A^{-n}\right\|<M$

Received by the editors August 4, 1978 and, in revised form, September 22, 1978.

AMS (MOS) subject classifications (1970). Primary 47A30, 47A65; Secondary 47B15. 
for $n>0$. In [2] it is shown that this condition implies that $A$ is similar to a scalar multiple of a unitary operator.

The converse assertion is clear.

In general one has $\mathscr{B}_{A} \supseteq\{A\}^{\prime}$, the commutant of $A$. In [2] it is shown that equality holds with $\operatorname{dim} \mathcal{H}<\infty$ if and only if $A$ is a nonzero scalar multiple of an operator of the form $1+N, N$ nilpotent. The necessity of this condition is also an immediate consequence of the next result.

LEMMA 1. Let $A$ be a bounded operator with $\mathscr{B}_{A}=\{A\}^{\prime}$. If $\lambda$ is an eigenvalue of $A, \bar{\mu}$ an eigenvalue of $A^{*}$, and if $|\lambda|<|\mu|$ then $\lambda=\mu$.

Proof. Suppose $f$ and $g$ are unit vectors with $A f=\lambda f, A^{*} \mu=\bar{\mu} g$. Then $A^{n}(f \otimes g) A^{-n}=\lambda^{n} \mu^{-n}(f \otimes g)$ is bounded for $n \geqslant 0$, hence $f \otimes g$ commutes with $A$ and this implies $\lambda=\mu$.

We now give a simpler proof that the condition $A=1+$ nilpotent is sufficient for $\mathscr{B}_{A}=\{A\}^{\prime}$. Observe that $A=1+Q^{\prime}$ with $Q^{\prime}$ nilpotent (quasinilpotent) if and only if $A=e^{Q}$ with $Q$ nilpotent (quasinilpotent). Moreover,

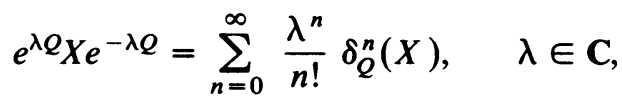

where $\delta_{Q}$ is the operator on $\mathscr{B}(\mathcal{H})$ given by $\delta_{Q}(X)=Q X-X Q$. Now if $Q$ is nilpotent so is $\delta_{Q}$, hence the entire function $e^{\lambda Q} X e^{-\lambda Q}$ reduces to a polynomial and is therefore bounded on the positive integers only if it is constant, that is, only if $\delta_{Q}(X)=0$ and $X \in\{Q\}^{\prime}=\{A\}^{\prime}$.

In the remainder of this paper we are concerned with sharpening the preceding argument to see to what extent $A=1+Q, Q$ quasinilpotent, is sufficient for $\mathscr{B}_{A}=\{A\}^{\prime}$.

LEMMA 2.

$$
\sup \left\{\left\|e^{t B} X e^{-t B}\right\|: t \geqslant 0\right\} \leqslant e^{2\|B\|} \sup \left\{\left\|e^{n B} X e^{-n B}\right\|: n=1,2, \ldots\right\}
$$

for any operators $X$ and $B$.

Proof. Each positive real number $t=n+r$ with $0 \leqslant r<1$, and $e^{r B}$ has norm at most $e^{\|B\|}$.

The preceding lemma gives another proof of the result of [2] that $\mathscr{B}_{A} \cap$ $\mathscr{B}_{A^{-1}}=\{A\}^{\prime}$ for $A$ positive and invertible. Write $A=e^{B}$ with $B$ Hermitian. If $X$ belongs to $\mathscr{B}_{A} \cap \mathscr{B}_{A^{-1}}$ and $\lambda=t+i$ then

$$
\sum_{0}^{\infty} \frac{\lambda^{n}}{n !} \delta_{B}^{n}(X)=e^{i s B} e^{t B} X e^{-t B} e^{-i s B}
$$

has norm at most $\sup \left\{\left\|e^{t B} X e^{-t B}\right\|: t \in \mathbf{R}\right\}<\infty$ so that, by Liouville's Theorem, the entire function on the left is constant and

$$
\delta_{B}(X)=0, \quad X \in\{B\}^{\prime} \subseteq\{A\}^{\prime} .
$$

In [2] it is conjectured that if $A=1+Q$ with $Q$ quasinilpotent then $\mathscr{B}_{A}=\{A\}^{\prime}$. We are unable to prove this. The next result, however, shows that $\mathscr{B}_{A} \cap \mathscr{B}_{A^{-1}}=\{A\}^{\prime}$.

THEOREM 2. If $Q$ is quasinilpotent and $X$ is an operator for which $\sup \left\{\left\|e^{n Q} X e^{-n Q}\right\|: n= \pm 1, \pm 2, \ldots\right\}<\infty$ then $Q X=X Q$. 
Proof. Let $g_{\phi}(z)=\phi\left(e^{z Q} X e^{-z Q}\right)$ for $\phi$ a linear functional of norm 1 on $\mathscr{B}(\mathcal{H})$. Then $g_{\phi}$ is an entire function of order

$$
\rho=\varlimsup_{r \rightarrow \infty} \frac{\log \log M_{\phi}(r)}{\log r} \leqslant \lim _{r \rightarrow \infty} \frac{\log 4\|Q\|+\log r}{\log r}=1 .
$$

(Here $M_{\phi}(r)$ is the maximum of $\left|g_{\phi}\right|$ on $|z| \leqslant r$.)

Suppose that $g=g_{\phi}$ has order $\rho<1$. If we choose $\alpha$ with $\rho<\alpha<1$ and $|g(z)|<K \exp \left(r^{\alpha}\right)$ for $|z|=r$ large enough. Let $f(z)=g(-i z)$. Then $|f(z)| \leqslant$ $C \exp \left(r^{\alpha}\right)$ for $z$ in the open right half-plane $H^{+}$. Also, $|f(i y)|<M<\infty$ for all real $y$ by Lemma 2 . It follows by a standard Phragmén-Lindelöf argument (see [3, p. 282]) that $|f(z)| \leqslant M$ in $H^{+}$. Thus $|g(z)|<M$ for $z$ in the lower half-plane. The same argument applied to $\overline{g(\bar{z})}$ shows that $g$ is bounded on $\mathbf{C}$. Therefore $0=g_{\phi}^{\prime}(0)=\phi(Q X-X Q)$.

It remains to consider the case in which $g_{\phi}$ has order 1 . In this case we claim that $g_{\phi}$ has type 0 . To prove this, let $\varepsilon>0$ and choose $N$ so that $\left\|\delta_{Q}^{n}\right\|<\varepsilon^{n}$ for $n>N$. Then

$$
\begin{aligned}
\left|g_{\phi}(z)\right| & \leqslant \sum_{n=0}^{N} \frac{r^{n}}{n !}\left|\phi\left(\delta_{Q}^{n}(X)\right)\right|+\sum_{n=N+1}^{\infty} \frac{r^{n}}{n !} \varepsilon^{n}\|X\| \\
& \leqslant\|X\|\left(K \cdot \sum_{0}^{N} r^{n}+e^{\varepsilon r}\right) \leqslant\|X\|(K+1) e^{e r}
\end{aligned}
$$

for $|z|=r$ large enough. It follows that the type $\tau$ of $g_{\phi}$ satisfies $\tau$ $=\varlimsup_{r}\left[\log M_{\phi}(r) / r\right] \leqslant \varepsilon$. Hence $\tau=0$ as $\varepsilon$ is arbitrary.

Therefore $g_{\phi}$ is of zero exponential type and is bounded on $\mathbf{Z}$, hence is constant (see [1, 10.2.11]).

We have $\phi(Q X-X Q)=0$ for all $\phi \in \mathscr{B}(\mathcal{H})^{*}$ and so $Q X-X Q=0$.

If $\mathcal{H}$ is finite-dimensional then $\mathscr{B}(\mathcal{H})=\mathscr{R}\left(\delta_{A}\right) \oplus\left\{A^{*}\right\}^{\prime}$ for any operator $A$, where the indicated orthogonality is with respect to the (trace) inner product on $\mathscr{B}(\mathcal{H})$. By considering $\mathscr{B}_{A}$ for $A=1+Q$ one obtains a characterization of $\{Q\}^{\prime}$, for $Q$ nilpotent, from the preceding results. It may be of interest to determine the corresponding characterization of the range of nilpotent and quasinilpotent derivations $\delta_{Q}$.

We conclude by mentioning that, as noted in [2], Theorem 2 implies that $\mathscr{B}_{A} \cap \mathscr{B}_{A^{-1}}=\{A\}^{\prime}$ for any operator $A$ that is quasisimilar to an operator of the form $1+Q, Q$ quasinilpotent. This fact would seem to discourage the search for a converse result. By certainly one can find a stronger necessary condition than that of the above lemma.

Note ADDED. After this paper was completed I learned that Paul Roth has found a quasinilpotent $Q$ such that $\mathscr{B}_{A} \neq\{A\}^{\prime}$ for $A=1+Q$.

\section{REFERENCES}

1. R. P. Boas, Jr., Entire functions, Academic Press, New York, 1954.

2. J. A. Deddens, Another description of nest algebras, preprint, 1978.

3. W. Rudin, Real and complex analysis, 2nd ed., McGraw-Hill, New York, 1974.

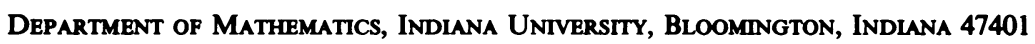

